\title{
Sampling and series expansion theorems for fractional Fourier and other transforms
}

\author{
Çag̃atay Candan ${ }^{\mathrm{a}, *, 1}$, Haldun M. Ozaktas ${ }^{\mathrm{b}, 2}$ \\ ${ }^{a}$ Department of Electrical Engineering, Middle East Technical University, TR-06531 Ankara, Turkey \\ ${ }^{\mathrm{b}}$ Department of Electrical Engineering, Bilkent University, TR-06533 Bilkent, Ankam, Turkey
}

Received 25 November 2002

\begin{abstract}
We present much briefer and more direct and transparent derivations of some sampling and series expansion relations for fractional Fourier and other transforms. In addition to the fractional Fourier transform, the method can also be applied to the Fresnel, Hartley, and scale transform and other relatives of the Fourier transform.
\end{abstract}

(C) 2003 Published by Elsevier B.V.

Keywords: Fractional transforms; Series expansion; Signal sampling

The fractional Fourier transform [10] is a generalization of the ordinary Fourier transform. It has received considerable interest over the past decade and has found many applications in optics and signal processing $[1,2,5-10]$. Of particular interest from a signal analysis perspective is the observation that as a signal is fractional Fourier transformed, its time- or space-frequency representations - such as the Wigner distribution-rotate in the time- or space-frequency plane. The fractional Fourier domains [6], which are generalizations of the conventional time/space and frequency domains, provide a continuous transition between the time/space and frequency domains.

A number of sampling and series expansion theorems for fractional Fourier transform have been derived [13-16]. Here we show how an elementary

\footnotetext{
* Corresponding author.

${ }^{1}$ Currently at School of ECE, Georgia Institute of Technology, Atlanta, USA.

${ }^{2}$ H.M. Ozaktas acknowledges partial support of the Turkish Academy of Sciences.
}

technique can reproduce these results in a much more direct way.

The fractional Fourier transform [10] of $f(t)$ with angle $\alpha$ is defined as ${ }^{3}$

$$
\begin{aligned}
& \mathscr{F}^{\alpha}\{f(t)\}\left(t_{\alpha}\right) \\
& =f_{\alpha}\left(t_{\alpha}\right)=\frac{K_{\alpha}}{\sqrt{2 \pi}} \mathrm{e}^{\mathrm{j}\left(t_{\alpha}^{2} / 2\right) \cot \alpha} \\
& \quad \times \mathscr{F}^{\pi / 2}\left\{\mathrm{e}^{\mathrm{j}\left(t^{2} / 2\right) \cot \alpha} f(t)\right\}\left(t_{\alpha} \csc \alpha\right),
\end{aligned}
$$

where $K_{\alpha}=\sqrt{(1-\mathrm{j} \cot \alpha)}$ and $\mathscr{F} \pi / 2$ is the ordinary Fourier transform operation, $\mathscr{F} \pi / 2\{f(t)\}(\omega)=F(\omega)=$ $1 / \sqrt{2 \pi} \int_{-\infty}^{\infty} f(t) \mathrm{e}^{-\mathrm{j} \omega t} \mathrm{~d} t$. The function $f_{\alpha}\left(t_{\alpha}\right)$ denotes the fractional domain representation of $f(t)$ with the rotation angle $\alpha$. Readers may examine [1,7] for the angle interpretation of the domain index. An extension of the continuous-input, continuous-output transform to discrete signals is given in $[3,4,11,12]$.

\footnotetext{
${ }^{3}$ We follow the notation of [13] which differs from $[7,10]$.
} 
Shannon's interpolation theorem for the ordinary Fourier transform expresses a band-limited function in terms of its time domain samples. It is possible to write the dual of this theorem for the time-limited functions. The dual theorem says that if $f(t)$ is time-limited to $[-T / 2, T / 2]$, the Fourier transform of $f(t)$ can be expressed as $F(\omega)=\sum_{n} F(n W) \operatorname{sinc}(\omega / W-n)$, where $W=2 \pi / T$.

To derive the sampling theorem for fractional Fourier transform, we define an intermediary function $v(t)=\mathrm{e}^{\mathrm{j}\left(t^{2} / 2\right) \cot \alpha} f(t)$. If $f(t)$ is time-limited, so is $v(t)$. The Fourier transform of $v(t)$ can then be calculated from the interpolation formula given in the preceding paragraph. By making use of this result, we can express the fractional Fourier transform of a time-limited function as

$$
\begin{aligned}
f_{\alpha}\left(t_{\alpha}\right)= & \frac{K_{\alpha}}{\sqrt{2 \pi}} \mathrm{e}^{\mathrm{j}\left(t_{\alpha}^{2} / 2\right) \cot \alpha} \sum_{n} V(n W) \\
& \times \operatorname{sinc}\left(\frac{t_{\alpha} \csc \alpha}{W}-n\right) .
\end{aligned}
$$

To eliminate $V(n W)$, we evaluate the expression above at $t_{\alpha}=m W \sin \alpha$ ( $m$ is an arbitrary integer). Upon this evaluation, we obtain a relation for $V(n W), K_{\alpha} / \sqrt{2 \pi} V(m W)=f_{\alpha}(m W \sin \alpha)$ $\times \mathrm{e}^{-\mathrm{j}\left((m W \sin \alpha)^{2} / 2\right) \cot \alpha}$. By substituting this relation in (2), we get the interpolation theorem of the fractional Fourier transform for the domain limited functions:

$$
\begin{aligned}
f_{\alpha}\left(t_{\alpha}\right) & \\
= & \mathrm{e}^{\mathrm{j}\left(t_{\alpha}^{2} / 2\right) \cot \alpha} \sum_{n} f_{\alpha}\left(\sin \alpha W_{n}\right) \\
& \quad \times \mathrm{e}^{-\mathrm{j}\left(\left(\sin \alpha W_{n}\right)^{2} / 2\right) \cot \alpha} \operatorname{sinc}\left(\frac{t_{\alpha} \csc \alpha}{W}-n\right) .
\end{aligned}
$$

This relation implies that a function limited at a fractional domain can be represented by its samples at any other fractional domain. This first fundamental relation is equivalent to expressions which have been previously presented by Xia [15] and Zayed [16].

Now, by applying the inverse transform $\mathscr{F}^{-\alpha}$ to both sides of (3); we immediately get the equivalent of the classical Fourier series for the fractional transform.

$$
\begin{aligned}
f(t)= & \sqrt{2 \pi} \frac{K_{-\alpha}|\sin \alpha|}{T} \sum_{n} f_{\alpha}\left(\sin \alpha W_{n}\right) \\
& \times \mathrm{e}^{-\mathrm{j}\left(t^{2}+\left(\sin \alpha W_{n}\right)^{2}\right)(\cot \alpha / 2)+\mathrm{j} n W t} .
\end{aligned}
$$

This second fundamental relation was presented by Pei et al. [13], but was arrived at a lengthier path.

The same technique can be applied to other transforms with a suitable intermediary function. We present another application on Cohen's scale transform [4]. The relation between the scale transform and Fourier transform is given by $\{\mathscr{S} f\}(c)=$ $\mathscr{F}\{\mathscr{W}\{f\}\}(c)$ where $\mathscr{W}$ is the exponential warping operation, $f^{W}(t)=\mathscr{W}\{f\}(t)=f\left(\mathrm{e}^{t}\right) \mathrm{e}^{t / 2}$. Assuming that $f(t)$ is scale-limited to $C_{0}$, it is possible to write an analogous series expansion in scale domain as

$f^{W}(t)=\sum_{n} f^{W}\left(\frac{n}{C_{0}}\right) \operatorname{sinc}\left(C_{0} t-n\right)$.

Applying the inverse warping operation, we obtain the sampling theorem for the scale transform, [4]

$$
\begin{aligned}
f(t) & =\mathscr{W}^{-1}\left\{f^{W}(t)\right\} \\
& =\sum_{n} f\left(e^{n / C_{0}}\right) e^{n / 2 C_{0}} \frac{\operatorname{sinc}\left(C_{0} \ln (t)-n\right)}{\sqrt{t}} .
\end{aligned}
$$

Another point of interest is the Parseval's relation for the domain limited functions. By taking the magnitude square of both sides of (4) and then integrating, we reach the Parseval's relation for the fractional Fourier series

$$
\int_{-T / 2}^{T / 2}|f(t)|^{2} \mathrm{~d} t=W|\sin \alpha| \sum_{n}\left|f_{\alpha}\left(\sin \alpha W_{n}\right)\right|^{2} .
$$

The reader may wish to examine following cases to gain more insight on the continuum of fractional domains: As $\alpha \rightarrow \pi / 2$, Eq. (7) evolves into classical Parseval's relation. As $\alpha \rightarrow 0$, the summation on the right side of (7) turns into the integration operation on the left, thus making both sides identical. As the span of the function $f(t)$ expands in time, that is $T \rightarrow \infty$; Eq. (7) reduces to the unitarity property of the continuous fractional Fourier transform. Similarly as $T \rightarrow \infty$, the fractional series expansion given in (4) approaches to the definition of fractional Fourier transform given in (1).

Although we do not provide further examples, the presented approach can be applied to many other transforms including Fresnel transform, Hartley transform and to the other relatives of Fourier transform.

In conclusion, we have presented a simple technique which allows briefer and more direct derivations of 
sampling and series expansions theorems for fractional Fourier and other transforms. Apart from representing simplification of the analysis of previous papers, the technique can be applied to a variety of transforms and should be useful as a generic tool which can produce key relations systematically and effortlessly in a few steps.

\section{References}

[1] L.B. Almeida, The fractional Fourier transform and time-frequency representations, IEEE Trans. Signal Process. 42 (1994) 3084-3091.

[2] L. Barker, Ç. Candan, T. Hakioğlu, M.A. Kutay, H.M. Ozaktas, The discrete harmonic oscillator, Harper's equation, and the discrete fractional Fourier transform, J. Phys. A 33 (2000) 2209-2222.

[3] Ç. Candan, M.A. Kutay, H.M. Ozaktas, The discrete fractional Fourier Transform, IEEE Trans. Signal Process. 48 (2000) 1329-1337.

[4] L. Cohen, The scale representation, IEEE Trans. Signal Process. 41 (1993) 3275-3292.

[5] M.A. Kutay, H. Özaktas, H.M. Ozaktas, O. Arıkan, The fractional Fourier domain decomposition, Signal Processing 77 (1999) 105-109.

[6] H.M. Ozaktas, O. Aytür, Fractional Fourier domains, Signal Processing 46 (1995) 119-124.
[7] H.M. Ozaktas, B. Barshan, D. Mendlovic, L. Onural, Convolution, filtering, and multiplexing in fractional Fourier domains and their relation to chirp and wavelet transforms, J. Opt. Soc. Am. A 11 (1994) 547-559.

[8] H.M. Ozaktas, M.F. Erden, Relationships among ray optical, Gaussian beam, and fractional Fourier transform descriptions of first-order optical systems, Opt. Commun. 143 (1997) $75-86$.

[9] H.M. Ozaktas, D. Mendlovic, Fractional Fourier optics, J. Opt. Soc. Am. A 12 (1995) 743-751.

[10] H.M. Ozaktas, Z. Zalevsky, M.A. Kutay, The Fractional Fourier Transform with Applications in Optics and Signal Processing, Wiley, New York, 2001.

[11] S.C. Pei, M.H. Yeh, Improved discrete fractional Fourier transform, Opt. Lett. 22 (1997) 1047-1049.

[12] S.C. Pei, M.H. Yeh, The discrete fractional cosine and sine transforms, IEEE Trans. Signal Process. 49 (2001) $1198-1207$.

[13] S.C. Pei, M.H. Yeh, T.L. Luo, Fractional Fourier series expansion for finite signals and dual extension to discrete-time fractional Fourier transform, IEEE Trans. Signal Process. 47 (1999) 2883-2888.

[14] H. Sundaram, S.D. Joshi, R.K.P. Bhatt, Scale periodicity and its sampling theorem, IEEE Trans. Signal Process. 45 (1997) $1862-1865$.

[15] X.G. Xia, On bandlimited signals with fractional Fourier transform, IEEE Signal Process. Lett. 3 (1996) 72-74.

[16] A.I. Zayed, On the relationship between the Fourier and fractional Fourier transforms, IEEE Signal Process. Lett. 3 (1996) 310-311. 